\title{
Improvementsin the performance of an incubation-type planar patch clamp biosensor usinga salt bridge electrodeand a plastic (PMMA) substrate
}

\author{
Hidetaka Uno $^{a, b}$, Zhi-hong Wang ${ }^{a, b}$, Ysutaka Nagaoka ${ }^{a, b}$, Noriko Takada ${ }^{c}$, \\ Senthilkumar Obuliraj ${ }^{a, b . d}$, Kei Kobayashi ${ }^{a, b}$, Toru Ishizuka ${ }^{b, e}$, \\ Hiromu Yawo ${ }^{b, e}$, Yukio Komatsu ${ }^{\mathrm{f}}$, Tsuneo Urisu ${ }^{a, b} *$
}

${ }^{a}$ FIRST Research Center for Innovative Nanobiodevices, Nagoya University, Furo-cho, Chikusa-Ku, Nagoya 464-8603, Japan

${ }^{b}$ JST, CREST,

K's Gobancho, 7 Gobancho, Chiyoda-ku, Tokyo 102-0076, Japan

${ }^{c}$ Institute for Molecular Science,

Myodaiji, Okazaki 444-8585, Japan

${ }^{d}$ Department of Chemistry, Ramco Institute of Technology, Rajapalayam, 626117,India

${ }^{e}$ Department of Developmental Biology and Neuroscience, Tohoku University Graduate School of Life Sciences,

2-1-1 Katahira, Aobaku, Sendai, 980-8577, Japan

${ }^{f}$ Research Institute of Environmental Medicine, Nagoya University,

Furo-cho, Chikusa-Ku, Nagoya 464-8603, Japan

*Corresponding author: FIRST Research Center for Innovative Nanobiodevices, Nagoya University, Furo-cho, Chikusa-Ku, Nagoya 464-8603, Japan

e-mail: t.urisu@nanobio.nagoya-u.ac.jp

Key words: planar patch clamp, salt bridge electrode, ion channel biosensor, high-throughput screening, Channelrhodopsin

(C) 2013. This manuscript version is made available under the Elsevier user license

http://www.elsevier.com/open-access/userlicense/1.0/ 


\begin{abstract}
Incubation-type planar patch clamp biosensors, first described in 2008, are expected to enable the realization of high-throughput neural network screening. Several technical challenges must be overcome before this technique may be implemented. First, it is difficult to achieve a sufficiently high seal resistance (a gigaohm seal), which is an important parameter for patch clamp operation. A low seal resistance produces large baseline fluctuations corresponding to the fluctuation of the solid-liquid and liquid-liquid interface potentials.Second, the sensor cell must be positioned at a micropore over a long incubation time while maintaining the cell viability. Finally, a low-cost high-performance sensor chip suitable for high-throughput screening must be developed. These problems have been resolved to some extent in the present work. A stable electrode with a salt bridge structure was developed here to reduce the baseline fluctuations. The cell positioning technology was improved by forming a novel cell trapping pattern using hot embossing on a plastic (polymethylmethacrylate (PMMA)) substrate for use in a microfluidic sensor chip.The combination of the stable electrode and the cell trapping pattern dramatically increased the device success probability from $\sim 2 \%$ to $60-80 \%$ using HEK293 cells as a model system. The utility of the present results was tested by characterizing rat hippocampal neuron networks. The seal resistance wasfound to be lower than the seal resistance obtainedfrom the HEK293 cells. The effectiveness of the stable electrodes, however, was significantly clear and spontaneous channel currents from the neural network were successfully observed.
\end{abstract}




\section{Introduction}

The pioneering studies of ionchannel biosensors were performed usingpipette patch clamptechniques [1-3]. The recently developed planar patch clamp offers the possibility of developing high-throughput screening applications[4-10]. The planar patch clamp methodhas several advantages over the classical pipette patch clamp in a high-throughput recording system: the former requires less equipment, is insensitive to mechanical vibrations, is capable of collecting long-term measurements, and is relatively easy to use. High-throughput screening devices based on planar patch clamps are currently commercially available [11]. Commercialized planar patch clamp devices, however, cannot be used in a system that requires long incubation times. The functional analysis and/or screening devices could be improved by introducing incubation capabilities to the planar patch clamp method. Such a technique would be especially useful in applications to in vitro neural network studiesinvolving dissociated cultured neurons [12-15]. We previously developed an incubation-type planar patch clamp device and demonstrated its operation using the transient receptor potential cation channel subfamily V1 (TRPV1)-expressing HEK293 cells with capsaicin as a ligand molecule [16, 17]. Several outstanding technical challenges, however, remain in the current incubation-type planar patch clamp biosensors.

A high seal resistanceis difficult to obtain using an incubation-type planar patch clamp. The seal resistance is typically in the range of a few megaohms to a few tens of megaohms, whereas seal resistancesexceeding $100 \mathrm{M} \Omega$ are easily obtained using conventional planar patch clamp methods [11, 18-20].This is considered to be quite reasonable if we remember the experience with the pipette patch clamp where the seal resistance significantly decreases due to contaminations by proteins and lipid molecules on the cell surface if the tip of the pipette comes into contact with the cell surface even once. So we think that, at least at the present stage, low seal resistance is the essential weak point of the incubation-type planar patch clamp, in which the extra cellular matrix (ECM) molecules between the sensor chip surface around the micropore and the sensor cell membrane easily decrease the seal characteristics. The analysis of two-dimensional networks of cultured neurons is considered to be one of the most important applications of the incubation-type planar patch clamp method.The seal resistance problem is much more significant in the context of neural networks, which require, in some cases, several weeks to several months of incubation time. The substrate-cell membrane gap may be contaminated by the debris from cells and proteinsin the nutrient medium or the secretionsof the neurons. The tensile forces among neighboring cells induced by the synapse formations increase the gap size or remove cells from the substrate surface. Despite these technical challenges, previous studies have not discussed the current noise caused by the low seal resistance, and no solutions to this problem have been offered.

Sensor cells are positioned on a micropore by suction in the conventional planar patch clamp method, and the channel currents are measured immediately after cell positioning. The incubation-type method, by contrast, requires the sensor cellsto be continuously positioned on the micropore for long incubation 
times, usually more than one week in the case of neural cells. Micro contact printing of the ECM may be used to induce a cell arrangement pattern [21].This alignment of the ECM pattern with the micropore in each experimentrequires the use of expensive equipment. Our tests of this technique resulted in the successful positioning of the sensor cells onto the micropores (unpublished results); however, printing failures, such as misaligned or blurred printing, were common. A more reliable and easier method is required.

In this work, we reduced the noise due to the low seal resistance by developing a stable salt bridge-type electrode and successfully applied this electrode in the incubation-type planar patch clamp method. An ionchannel biosensor was constructed using TRPV1-expressing HEK293 cells as the sensor cells, and excellent performance was confirmed by measuring the capsaicin-gated channel current. Anovel plastic (polymethylmethacrylate (PMMA)) sensor chip bearing a cell trapping pattern and a microfluidic structure was developed using hot embossingtechniques [22]. This new cell positioning method replaced the micro contact printing approach. The recently developed light-gated ion channel method provides an alternative approach to preparing ion channel biosensor devices for performance tests. This method offers excellent temporal and spatial resolution and is easyto implement [23-30]. This PMMA sensor chip was used in the present ion channel biosensor,yielding a performance that was equivalent to that of the pipette patch clamp in the whole-cell mode, as confirmed by measuring the laser-induced channel current of the channel rhodopsin wide receiver (ChRWR) [27] expressed on HEK293 sensor cells. A combination of these two technologies, the stable electrode and the cell trapping pattern, dramatically increased the probability of success (that is, the number of devices in which reliable channel current profiles were obtained relative to the total number of fabricated devices) from $\sim 2 \%$ to $60-80 \%$ using HEK293 cells as a model system. The importance of the stable electrode was further supported by successfully recording the spontaneous ion channel currents from the in vitro neural network formed from dissociated neurons derived from rat hippocampal tissue.

\section{Materials and Methods}

\subsection{Ion channel Biosensor and Stable Electrode}

Figure 1(a) shows a schematic structure of the ion channel biosensor used in this work. The sensor chip was sandwiched between the upper (bath solution side) and the lower (pipette solution side)polydimethylsiloxane (PDMS) plates. Microfluidic circuits were formed inside of the lower PDMS plate to supply and exhaust the pipette solutions. The bath solutions were supplied or exhausted through polytetrafluoroethylene (PTFE) tubes.The stable electrodes of salt-bridge structure (Fig. 2 (a))were used both for the bath solution side as a ground and the pipette solution side as a membrane voltage applied 
electrode.The $\mathrm{AgCl} / \mathrm{Ag}$ wire of the stable electrode, which was formed by painting $\mathrm{AgCl}$ ink (BAS Inc.) onto the surface of the $\mathrm{Ag}$ wire $(0.3 \mathrm{~mm}$ diameter $)$, was inserted into a Pyrex glass tube filled with a saturated $\mathrm{KCl}$ and $\mathrm{AgCl}$ solution,and the tip was sealed with Vycor glass (GIKENKAGAKU Co. Ltd.). The $\mathrm{AgCl} / \mathrm{Ag}$ wire was connected to a gold-plated electrode pin, as shown in Fig. 2(a).

\subsection{Si and PMMA sensor chips}

The Si sensor chip fabrication process,involving the preparation of a Si-on-insulator (SOI) substrate, was reported previously[16, 17]. A thermal oxidation layer about $1 \mu \mathrm{min}$ thickness was formed on the substrate surface by wet thermal oxidation at $900^{\circ} \mathrm{C}$ using a water-saturated $\mathrm{O}_{2}$ flow. A large well on the reverse surface was subsequently made using a diamond drill. A pyramid-shaped hole that reached the buried $\mathrm{SiO}_{2}$ layer was formed by etching in $8 \%(\mathrm{v} / \mathrm{v})$ tetramethylammonium hydroxide at $90^{\circ} \mathrm{C}$ for about $40 \mathrm{~min}$. A micropore with a diameter of 1-2 $\mu \mathrm{m}$ was formed in the silicon membrane by applying a focused ion beam (FIB, Seiko Instruments Inc.) to the reverse side, as shown in Fig. 3(a).

We developed a novel sensor chip using a PMMA substrate (PMMA sensor chip), the structure of which is shown in Figs. 3(b),(c),(d). A cell trapping pattern, as shown in Figs. 3 (b),(c),(d), was formedon the bath-solution-side surface of the PMMA sensor chip. On the other surface of the PMMA sensor chip, a pipette solution well was formed, as shown in Fig. 3(b). These structures were formed using a two-sided hot embossing machine (Engineering System Co. Ltd.) on a $0.2 \mathrm{~mm}$ thick PMMA substrate. Ni andbrass molds were used for the bath andpipettesolutionside patterning processes, respectively. The Ni mold used for the cell trapping pattern formation was formed by first preparing the resist pattern via photolithography using a positive resist AZP4903 (AZ Electronic Materials Ltd.), which wasapplied the mirror-polished surface of the $40 \mathrm{~mm}$ x $40 \mathrm{~mm}$ square and $0.6 \mathrm{~mm}$ thick $\mathrm{Si}(100)$ substrate. The surface of the Si substrate was exposed to hexamethyldisilazane (Tokyo Chemical Industry Co., Ltd.) vapor to improve the adherence of the photoresist to the Si surface. The Ni mold was formed by electroforming (IKEX Industry Co., Ltd.) using this resist pattern as the master mold. The depth of the cell trapping pattern was $8-10 \mu \mathrm{m}$.

The brass mold used for to prepare the pipette solution well was formed using ultrafine machinery (FANUC Ltd.). The thickness of the thin film region between the cell trapping round area and the pipette solution well was about $10 \mu \mathrm{m}$ (Fig. 3(b)). The micropore having a diameter of $1.5 \mu \mathrm{m}$ to $2 \mu \mathrm{m}$ was formed at the center of the round area of the cell trapping pattern using FIB milling (Figs. 3(d)). The acceleration voltage and the current of the Ga ion beam were $30 \mathrm{kV}$ and $50 \mathrm{pA}$, respectively. Prior to FIB milling, a $\mathrm{Cu}$ thin film was deposited on both surfaces of the PMMA substrate to reduce the charge-up effects during FIB processing. This $\mathrm{Cu}$ thin film was easily removed using a dilute $\mathrm{HNO}_{3}$ solution after micropore formation. 


\subsection{Expression of ChRWR and TRPV1 on HEK293}

The chimeric molecule ChR(ABCDEfg), denoted "ChRWR", and its plasmid construct are described in ref. 27. HEK293 cells, which were a generous gift from Minoru Wakamori of the Tohoku University, were cultured at $37^{\circ} \mathrm{C}$ and $5 \% \mathrm{CO}_{2}$ in Dulbecco's modified Eagle's medium (DMEM) (Sigma-Aldrich Co.) supplemented with $10 \%$ fetal bovine serum (FBS, Biological Industries Ltd).The cells were transfected using the Effectene transfection reagent (Qiagen, Tokyo, Japan) according to the manufacturer's protocol. After cloning twice with the addition of G418 (Gibco) in a $10 \mathrm{~cm}$ dish, single colonies identified using bright Venus [31] fluorescence were selected using a cloning cylinder IWAKITE-32 (Asahi Glass Co., Ltd.) and cultured in a medium containing G418 until confluencehad been reached. TRPV1-expressing HEK293 was the gift from Professor Makoto Tominaga of the Okazaki Institute for Integrative Bioscience. Details of the transfection procedure are reported in ref. 32.

\subsection{Culture of HEK293 cells in the biosensor}

The surfaces of both the Si and PMMA sensor chips were coated with poly-L-lysineECM (PLL, Sigma-Aldrich Co.), which performed better in the preliminary biosensor culture experiments than the fibronectin ECM used previously[16, 17].The $50 \mu \mathrm{L}$ solution was dropped onto the substrate surface, followed by incubation for 1-2 days at room temperature. At this stage, the surface densities of the ECM were about $3-5 \mu \mathrm{g} / \mathrm{cm}^{2}$. After removal of excess solution, the substrate was rinsed with sterilized water, dried under a gentle nitrogen stream, and kept sterile until use. Cells were cultured in dishes filled with the medium under conventional incubating conditions: $37^{\circ} \mathrm{C}$ and $5 \% \mathrm{CO}_{2}$. The culture medium was supplemented with DMEM to which were added 10\% (v/v) FBS, 1\% (v/v) GlutamaxTM (Gibco), and $0.5 \%(\mathrm{v} / \mathrm{v})$ penicillin/streptomycin (Gibco). After the cells had been detached from the culture dishes, the cell suspension was seeded at a density of $100-300$ cells $/ \mathrm{mm}^{2}$ onto the ECM-coated chip. The channel current was measured after 5 days of culturing. At this point, about $70 \%$ confluence had been reached on the Si chip prepared without a cell trapping pattern, as shown in Fig. 4(a). The PMMA sensor chipprepared with a cell trapping pattern yielded a channel current measurement after about 3 days of culturing, once the monolayer colony around the micropore had been formed (Fig. 4(b)).

\subsection{Formation of a rat hippocampal neuron network on the Si sensor chip}

Rat embryos were obtained at embryonic day 17by caesarian section from pregnant mothers, which were anaesthetized with isoflurane (Abbott)and sacrificed by scission of the carotid. The use of these animals in the experimental protocols was approved by theExperimental Animal Committee of the 
Research Institute of Environmental Medicine, Nagoya University. The brains of 8-10 embryos were quickly removed using surgical scissors, and the hippocampal tissue was dissected into small pieces that were subsequently transferred into a $15 \mathrm{~mL}$ plastic centrifuge tube with $5 \mathrm{~mL}$ Hank's Balanced Salt Solution (HBSS, Gibco) containing 0.25\% trypsin. The cells were then maintained at $37^{\circ} \mathrm{C}$ for $20 \mathrm{~min}$ in a water bath. HBSS was removed and the neural cells were disassembled by pipetting in $5 \mathrm{~mL}$ D-MEM with $10 \%$ fetal bovine serum (FBS). The tube was centrifuged at $140 \mathrm{~g}$ for $5 \mathrm{~min}$, and the cell pellet was suspended in1 mL Neurobasal medium containing B27 (Gibco), 2 mM GlutaMAX (Gibco) and 5\% FBS. The cells were plated at a density of $1-5 \times 10^{4}$ cells $/ \mathrm{cm}^{2}$ onto a PLL-coated Si sensor chip of $11 \mathrm{~mm}^{2}$ in $35 \mathrm{~mm}$ plastic culture dishes. The cultures were maintained at $37^{\circ} \mathrm{C}$ in a humidified atmosphere containing $5 \% \mathrm{CO}_{2}$ for 10-14 days. After the formation of the neuron network on the Si sensor chip surface, the biosensor chip was configured for the channel current measurements.

\subsection{Measurement of the ion channel current}

The electrical measurement systems used here were the same as those described in our previous planar patch clamp experiments $[16,17]$. An ion channel biosensor and preamplifier head stage were positioned inside the aluminum electromagnetic shield box. The culture medium was replaced with the bath solution and the pipette solutions which were added to the upper and lower chambers, respectively. The bath solution in the upper chamber contained $140 \mathrm{mM} \mathrm{NaCl}, 3 \mathrm{mM} \mathrm{KCl}, 10 \mathrm{mM}$ HEPES, $2.5 \mathrm{mM} \mathrm{CaCl}_{2}, 1.25$ $\mathrm{mM} \mathrm{MgCl} 2$, and $10 \mathrm{mM}$ glucose at $\mathrm{pH} 7.4$ (with $\mathrm{HCl}$ ). The lower chamber solution (pipette solution) contained: $40 \mathrm{mM} \mathrm{CsCl}, 80 \mathrm{mM} \mathrm{CsCH}_{3} \mathrm{SO}_{4}, 1 \mathrm{mM} \mathrm{MgCl}_{2}, 10 \mathrm{mM}$ HEPES, $2.5 \mathrm{mM} \mathrm{MgATP}$, and $0.2 \mathrm{mM}$ $\mathrm{Na}_{2}$ EGTA (pH 7.4). All data were recorded using a patch-clamp amplifier (Axonpatch 200B) at room temperature. Data were obtained using a $1 \mathrm{kHz}$ or $2 \mathrm{kHz}$ low-pass filter and an output gain of $1 \mathrm{mV} / \mathrm{pA}$. The data were then analyzed using the pClamp 9.2 software. Whole-cell current recordings were conducted by forming sub-nanometer conductive pores through the cell membrane, which electrically connected the inside of the cell to the lower chamber, upon the application of nystatin (Sigma) solutions to the lower chamber [33]. The nystatin stock solution was prepared by dissolving nystatin in $1 \mathrm{~mL}$ methanol and successively adding $45 \mu \mathrm{L} \mathrm{HCl}(1 \mathrm{M})$ and $45 \mu \mathrm{L} \mathrm{NaOH}(1 \mathrm{M})$. This solution was then diluted with the lower chamber solution to a final concentration of $100-200 \mu \mathrm{g} / \mathrm{mL}$ prior to use. The preparation of the whole-cell arrangement was confirmed by observing a capacitance increase of about 10 $\mathrm{pF}$ over 5-10 min after the addition of the nystatin solution to the lower chamber.

The laser-induced channel current measurements were collected by removing the inlet and outlet tubes of the bath solution side shown in Fig. 1. The laser beam from a $473 \mathrm{~nm}$ semiconductor laser with a 3.2 $\mathrm{mW}$ maximum output power (Sumitomo Osaka Cement Co., Ltd) was guided through an optical fiber and focused with a $26.5 \mathrm{~mm}$ focal length microlens under the fluorescence microscope's objective lens (Olympus). The beam diameter at the focal point was 30-100 $\mu \mathrm{m}$. 


\section{Results and discussion}

\subsection{Noise properties of the incubation-type planar patch clamp}

$\mathrm{AgCl} / \mathrm{Ag}$ electrodes are frequently used in patch clamp experiments. The simple $\mathrm{AgCl} / \mathrm{Ag}$ wire electrodes underwent a reaction at the interface between the $\mathrm{AgCl} / \mathrm{Ag}$ wire surface and the buffer solution: $\mathrm{Ag}+\mathrm{Cl}^{-} \leftrightarrow \mathrm{AgCl}+$ e-. The interface structure changes over time not only due to this chemical reaction, but also the uncontrollable contamination from the compounds or ions in the solution under the interface potential which obeys Nernst equation and the biased membrane potential.These interface structure alterations cause the electrode potential fluctuations. The electrode potential easily fluctuatesalso if the interface is disturbed by adding a solution of the ligand molecule.The electrode potentialinitially driftsuntil the interface structure becomesstationary [34].The solution surface level fluctuations, that is, the fluctuationsin the immersions depth of the $\mathrm{AgCl} / \mathrm{Ag}$ wire, also produced electrode potential fluctuations [34]. The simple $\mathrm{AgCl} / \mathrm{Ag}$ wire electrode yieldedbase line fluctuations and/or drift several tens to hundredsof pA at a $10 \mathrm{M} \Omega$ seal resistance, as shown in Fig. 2(b).

Thechange in the electrode potential ( several $\mathrm{mV}$ )isnot significant if the seal resistance is sufficiently high (gigaohm seal). In the case of the incubation-type planar patch clamp, however, if the seal resistance is assumed to be $10 \mathrm{M} \Omega$, a potential change of several millivolts produces a baseline change of several hundreds of $\mathrm{pA}$, comparable to or larger than the signal current. To overcome the electrode potential fluctuations, a salt bridge electrode structure was used in subsequent experiments, as shown in Fig. 2(a) $[35,36]$.

The low seal resistance typically observed inincubation-type planar patch clampsmay potentially be overcome by reducing the electrode potential fluctuations using a saltbridge-stabilized electrode because the dominant measurement mode in high-throughput screening and typical electrophysiology applications is the whole-cell mode, in which the channel current level exceeds several tens of pA.

The noise properties of the present biosensor were measured inside a shield box. The baseline fluctuations produced using the stable electrode were found to decrease significantly, as shown in Fig. 2(b). The observed total current noise $I_{t}(\mathrm{rms})$ obtained when using the stable electrode (Fig.2(a) lower trace) was typically $\sim 1.6 \mathrm{pA}$. This value was close to the thermal noise $I_{R}=1.5 \mathrm{pA}$ (rms value)calculated using [37]

$$
I_{R}^{2}=4 k T B / R_{j}
$$

where $k$ is the Boltzmann constant $\left(k=1.38 \times 10^{-23} J^{-1}\right), B(=2 \mathrm{kHz})$ is the frequency bandwidth of the circuit, $R_{j}(15 \mathrm{M} \Omega)$ is the seal resistance, and $T$ is the absolute temperature. 
The present results suggest that a several-fold increase in the seal resistance, which can reduce both the thermal noise and the electromagnetic induction noise, is extremely effective in reducing the total current noise.

\subsection{Application of the stable electrodes to an incubation-type planar patch clamp biosensor using HEK293cells}

The utility of the stable electrodes described here was demonstrated byapplying the stable electrodes to an incubation-type planar patch clamp biosensor constructed using established sensor cell and ion channelsystems, including TRPV1-expressing HEK293 [10, 17 ] and ChRWR-expressing HEK293 [38].

\subsubsection{Detection of capsaicin by the TRPV1-expressing HEK293 biosensor}

An ion channel biosensor should ideally be widely applicable in a variety of applications ranging from the detection of biological warfare agents to high-throughput screening devices for pharmaceutical development.Such biosensors should display an extremely high selectivity and sensitivity. In this work, we constructed the single-channel biosensor shown in Fig. 1 usinga Si or PMMA sensor chip. We first constructedan ion channel biosensor using TRPV1-expressing HEK293 cells for the detection of capsaicin, the main ingredient in hot chili peppers.Capsaicin elicits the sensation of a burning pain by selectively activating sensory neurons. Capsaicin stimulation opens the TRPV1 ion channel and nonselectively inducescation flow through the channel. The presence of $\mathrm{Ca}^{2+}$ in the extracellular solution rendersTRPV1 insensitive to capsaicinunder prolonged exposure. The mechanism underlying these effects has not yet been elucidated [32]. The increase in the intracellular $\mathrm{Ca}^{2+}$ concentration mediates the desensitizationresponse, and a variety of signaling pathwaysare implicated in the desensitization of TRPV1[32, 39].

The sensitivity to capsaicin was investigated by replacing the bath solution (without capsaicin)with a solution containing certain concentrations of capsaicin using a syringe pump (KD Scientific Inc.) at a pumping speed of $12 \mathrm{~mL} / \mathrm{h}$. The measurements were carried out at room temperature. An inward current, followed by desensitization, was observed in the presence of $\mathrm{Ca}^{2+}$ ions in the bath solution, Fig. 5(a).Desensitization was not observed in the $\mathrm{Ca}^{2+}$-free bath solution, as shown in Fig. 5(a), in good agreement with the reported measurements obtained using a pipette patch clamp [32]. The observed dependence of the peak current on the capsaicin concentration is shown in Fig. 5(b). The lower detection limit was about $0.01 \mu \mathrm{M}$. The highly selective and sensitive detection of neurotransmitter molecules and biologically active molecules by the ion channel biosensor using a pipette patch clamp has been reportedpreviously [1-3]. In those studies, the noise level of the baseline fluctuations was 5-20 pA (p-p). 
The noise level of 10-20 pA (p-p) observed in the present case (Fig. 5(b)) revealed that an ion channel biosensor having a performance nearly equivalent to that of the pipette patch clamp could be constructed using an incubation-type planar patch clamp equipped with a saltbridge stable electrode. These experiments were performed in the whole-cell mode. In this experiment, the use of a stable electrode was essential. The success probability increased from 1-2\% (simple $\mathrm{AgCl} / \mathrm{Ag}$ electrode) to about $60 \%$ (stable electrode).

3.3. Laser-induced channel current upon excitation of the ChRWR-expressing HEK293 cells, anda comparison between the Si and PMMA sensor chips

Although the Si (SOI) substrate displayed several advantages, the PMMA substrate appeared to be more attractive for sensor applications based on high-throughput screening devices using an incubation-type planar patch clamp. The SOI substrate readily permitted the precise microfabrication of a nanometer-level flat surface suitable for achieving a high seal resistance. Integration with the electrical circuit was easy. On the other hand, the PMMA substrate was low-cost, readily microfabricated using hot embossing techniques, and yielded a transparency that permitted observation using an inverse-type optical microscope, which is especially useful for cell imaging.

The performance of the ion channel biosensor based on a PMMA sensor chip was evaluated by measuring the laser-induced channel current of ChRWR-expressing HEK293 cells. A $20 \mu \mathrm{L}$ aliquot of the cell suspension $\left(1 \times 10^{4}\right.$ cells $\left./ \mathrm{mL}\right)$ was injected in the cell trapping area and incubated for 3 days. After the formation of a single monolayer colony covering the micropore region had been confirmed (Fig. 4(b)), a nystatin solution (100-200 $\mu \mathrm{g} / \mathrm{mL}$ ) was prepared using the pipette solution, and the preparation of the whole-cell measurement mode conditions was confirmed by the measurement of a capacitance increase of about $10 \mathrm{pF}$ due to the nystatin perforation. After 3 days of incubation, the seal resistance of 7.4 $\mathrm{M} \Omega$ increased to9.4 M Uunder a negative pressure $(10 \mathrm{kPa})$ applied to the pipette solution. Control experiments were carried out in parallel. No channel currents were observed for cells that did not express ChRWR. The dependence of the channel current profile on the membrane potential upon irradiation of the ChRWR-expressing HEK293 cells with a rectangular pulse shapeis shown in Fig. 6(a). Similar channel current profiles wereobserved using the Si sensor chip. The cell trapping pattern was formed on the Si chip surface using photolithography techniques with a SU8 negative resist [38]. The channel current profiles on the PMMA sensor chip agreed well with the reported profile (ABCDEfg in Fig. 6A of ref. 27) measured using the pipette patch clamp at the important points unique to ChRWR [27].Almost no desensitization in the current profile was observed relative to the sharp on profile $(\sim 10 \mathrm{~ms})$ and the slow off profile $(\sim 20$ $\mathrm{ms})$.

Many studies have examined the molecular mechanisms underlying the on and off profiles of the channel current induced by the photo-excitation of channel rhodopsin [22,24,26,27]. Many aspects of this 
mechanism have remained unclear. Several widely accepted arguments against the photoexcitation mechanismand the subsequent relaxation include: the channel rhodopsin contains a retinal moiety that covalently bound to the apoprotein;the photoisomerization of the all-trans-retinal to the 13-cis configuration iscoupled to the protein conformational changes and the ion permeation[27].Many $(\sim 8)$ intermediate states, including the dark states,which have a lifetime longerthan several seconds,areobserved.Several photocycle models have been proposed [22,27,40]. The "On" transition time to the initial opening state was about $2 \mathrm{~ms}$, and the decay in the photocurrent upon turning off the light strongly dependson the intracellular $\mathrm{pH}$ [22]. The wild-type channel rhodopsin 2 (ChR2)wasexcited to the first channel open state by blue $(\sim 480 \mathrm{~nm})$ light over an irradiation transition time of about $2 \mathrm{~ms}$. The ChR2 was subsequently rapidly desensitized to the second channel open state. This state then decayed to the dark state via switching off of the irradiation light over a transition time of about15 ms [24]. The ChR1 photocurrent was only slightly desensitized during bright light illumination,whereas the ChR2 photocurrent was rapidly desensitized $[22,27]$. The $\mathrm{N}$ terminal segments of ChR2 were replaced with the homologous counterparts of $\mathrm{ChR} 1$, and several chimeras were generated having different current profiles [27]. ChRWR yielded asmall desensitization and a nearly flat and quite large current profile [27].

Theon profile in the Si sensor chip was moderately slow (Fig. 4 in ref. 38), possibly due to the large capacitance $(\sim 100 \mathrm{pF})$ of the Si sensor chip compared with the PMMA chip (3-5 pF). These data indicated that a highly reliable current profile equivalent to the profile obtained using the pipette patch clamp method could be obtained using the PMMA sensor chips together with the stable saltbridge electrodes. Without the cell trapping pattern, a long incubation time ( $>5$ days) was needed to stabilize the micropore coverage in the sensor cell (Fig. 4 (a)). Multiple layers of the sensor cell often formed during this long incubation time,making current measurements impossible. In the presence of the cell trapping pattern, a single monolayer colony formed and stably covered the micropore over a short incubation time

(Fig. 4 (b)). The cell trapping pattern both shortened the required incubation time ( $\sim 3$ days) and improved the success probability by $10-20 \%$.

\subsection{Recordings of the spontaneous ionchannel currents in neural networks}

We are especially interested in the spontaneous ionchannel currents (SICs) induced by the spontaneous firing and/or spontaneous release of the neurotransmitter molecules from the presynaptic membrane [12], since SICs are considered to contain the information with unique characteristics of the neural network. On the other hand, it is known that the neural network structure is changed by the external stimulations [41]. In this work, we have succeeded in recording SICs by the planar patch clamp for the first time by using the stable electrode developed in this work. SICs are the noise containing information with unique characteristics of the neural network. A new characterization method of neural networks can be developed by the recording of SICs by planar patch clamp which enables multipoint and long term measurements. 
One of the most important applications of the incubation-type planar patch clamps is the multi-point ion channel current recordings of neural networks. The stable electrode is essential and important to this case because a high seal resistance is particularly difficult to realize due to the very long incubation times. We attempted to form neural networks using a PMMA sensor chip prepared with a cell trapping pattern, as shown in Fig.3. It was difficult, however,to form a long-term stable neural network. The viability of the neuronal cells within the cell trapping area was much lower than the viability outside of the area (Fig. S1). Stable incubation inside of the cell trapping area was observed using PC12 and HEK293 cells. On the other hand, nearly all neurons in the primary culture of neurons, whichformeda network with a huge number of synapses, had diedif they were unable to form synapses with surrounding neuronsover the course of one week. The cell trapping pattern shown in Fig. 3, inside of which neurons could not extend neurites freely,was found to be unsuitable for maintaining a neural network over long culture periods. So in this experiment, we seeded rathippocampal neurons on the Si (SOI) sensor chip prepared with a flat surface to a density of $1-5 \times 10^{4}$ cells $/ \mathrm{cm}^{2}$ and incubated the culturein the $35 \mathrm{~mm}$ dish over 10-14 days. In all sensor chips,an inhomogeneous gathering of neurons as shown in Fig. 7a was observed, due tocell migration[42].In 1-2 chips out of every 10, a neural cell was found to have grown on a micropore on the chip. The seal resistance was surprisingly small, $2.7 \mathrm{M} \Omega$, prior to applying suction, and $3.4 \mathrm{M} \Omega$ after suction $(n=5)$. The sensor chip with a cell prepared with a micropore was set up at the sensor chip position in the ionchannel biosensor, as shown in Fig. 1.The upper and lower volumes were filled with bath and pipette solutions, respectively.In this case, the inlet and outlet tubes on the bath solution side (Fig. 1) were removed. The networkformed was very complex. A large number of multi-synaptic contacts and autaptic contacts [43]were formed. We considered in this work that the spontaneous channel currentmeasurementswould bemore important than those of the currents induced by the artificial stimulation, since the network structureswould become much morecomplex due to the synaptic plasticity induced by the artificial stimulation [44]. Prior to the nystatin perforation (the whole cell arrangement), no channel currents were observed under the $-30 \mathrm{mV}$ voltage clamp (Fig. 7b).Immediately after the injection of nystatin into the pipette solution, however, a highly reproducible channel current was measured that dependedon the membrane voltage, as shown in Fig. 7b. These signals were clearly due to the spontaneous activity of the neural network. The addition of tetrodotoxin (TTX), a typical antagonist of $\mathrm{Na}^{+}$channels [45], produced some unique changes in the channel current profile.The observed channelcurrents were thought to be a mixture of many postsynaptic currents induced by the spontaneous firing of the presynaptic neurons and the post-synapse currents induced by the spontaneous neurotransmitter release which comprised the miniature excitatory postsynaptic currents (mEPSC) and the miniatureinhibitory postsynaptic currents (mIPSC) [45]. The network was too complex to permit a detailed analysis of the channel current data. It is, however, clear that the stable electrodes permitted measurement of the spontaneous channel currents. The inhomogeneous gathering of neurons as a result of migrationprevented the collection of meaningful multipoint measurements; therefore, we are now 
developinga new type of cell trapping pattern and cell seeding technique which hindercell migration and enable the formation of more homogeneous neural networks. We are also planningto improve the seal resistance by adopting an hourglass shape [7]in the micropore structures.

\section{Conclusion}

In an attempt to overcome the weaknesses of the incubation-type planar patch clamp, that is, the difficultiesassociated with preparing a gigaohm seal, we developed a salt bridge-type stable electrode. This electrode dramatically reduced the noise properties, especially the baseline fluctuations, to a level almost equivalent to the levels typically obtained using the pipette patch clamp method in the whole-cell mode. The probability of a successful measurement was significantly improved from $1-2 \%$ to 60-80\%using HEK293 cells. An ion channel biosensor was constructed using TRPV1-expressing HEK293 cellsthat could detect capsaicin. An excellent performance, equivalent to the performance of a pipette patch clamp, was obtained using the stable electrode.

The stable positioning of the sensor cell on the micropore over long incubation timesoffered another important challenge for the incubation-type planar patch clamp. This problemwas addressed by developing the PMMA sensor chip using a cell trapping pattern with a microfluidic structure on the bath solution side surface. This cell trapping pattern not only shortened the incubation time required for the sensor cell to stably cover the micropore, the pattern improved the probability of success by $10-20 \%$. The PMMA substrate includes several attractive features: it is low in cost and easy to produce via microfabrication. These are important characteristics for multichannel high-throughput screening device applications. The small capacitance of the substrate provideda high-speed response time and good transparency enabled observation using an inverse optical microscope, which was especially useful for cell imaging. We measured the laser-induced channel currents of a ChRWR-expressing HEK293 sensor cell. The waveform unique to ChRWR and its dependence on the membrane potential agreed well with the values obtained under similar conditions using a pipette patch clamp. The stable electrodes were used to measure the spontaneousion channel currents produced by the in vitro networks of rat hippocampal neurons. Although the seal resistances were lower than those obtained from HEK293 cells, a stable and highly reproducible channel current was observed, indicating the importance of the stable electrode.

\section{Acknowledgments}

We thank the staff at the Equipment Development Center in the Institute for Molecular Science (IMS), 
especially Mitsukazu Suzui, Masaki Aoyama, and Nobuo Mizutani, for their help in making the device. This work was supported by the IMS special research projects, and by the Japan Science and Technology Agency Core Research for Evolutional Science and Technology (JST-CREST). 


\section{References}

[1] E. A. Schwartz, Depolarization without calcium can release gamma-aminobutyric acid from a retinal neuron, Science 238 (1987) 350-355.

[2] Y. Kataoka, H. Ohmori, Activation of glutamate receptors in response to membrane depolarization of hair cells isolated from chick cochlea, J. Physiol. 477 (1994) 403-414.

[3] A. Hazama, S. Hayashi, Y. Okada, Cell surface measurements of ATP release from single pancreatic beta cells using a novel biosensor technique, Pflugers Arch.437 (1998) 31-35.

[4] N. Fertig, R. H. Blick, J. C. Behrends, Whole cell patch clamp recording performed on a planar glass chip, Biophys. J. 82(2002) 3056-3062.

[5] A. Stett, C. Burkhardt, U. Weber, P. V. Stiphout, T. Knott, CYTOCENTERING: a novel technique enabling automated cell-by-cell patch clamping with the CYTOPATCH chip, Receptors Channels 9 (2003) 59-66.

[6] R. Pantoja, J. M. Nagarah, D. M. Starace, N. A. Melosh, R. Blunck, F. Bezanilla, J. R. Heath, Silicon chip-based patch-clamp electrodes integrated with PDMS microfluidics, Biosens. Bioelectron. 20(2004) 509-517.

[7] T. Sordel, S. Garnier-Raveaud, F. Sauter, C. Pudda, F. Marcel, M. De Waard, C. Arnoult, M. Vivaudou, F. Chatelain, N. Picollet-D'hahan, Hourglass $\mathrm{SiO}_{2}$ coating increases the performance of planar patch-clamp,J. Biotechnol. 125(2006) 142-154.

[8] B. Matthews, J. W. Judy, Design and fabrication of a micromachined planar patch-clamp substrate with integrated microfluidics for single-cell measurements, J. Microelectromech. Syst. 15 (2006) 214-222.

[9] X. Li, K. G. Klemic, M. A. Reed, F. J. Sigworth, Microfluidic system for planar patch clamp electrode arrays, Nano Lett. 6(2006) 815-819.

[10] Z. L. Zhang, T. Asano, H. Uno, R. Tero, M. Suzui, S. Nakao, T. Kaito, K. Shibasaki, M. Tominaga, Y. Utsumi, Y. L. Gao, T. Urisu, Fabrication of Si-based planar type patch clamp biosensor using silicon on insulator substrate, Thin Solid Films 516(2008) 2813-2815.

[11] J. Dunlop, M. Bowlby, R. Peri, D. Vasilyev, R. Arias, High-throughput electrophysiology: an emerging paradigm for ion channel screening and physiology, Nat. Rev. Drug Discov. 7 (2008) 358-368.

[12] Wu Ma, Qi-Ying Liu, David Jung, Patricia Manos, Joseph J. Pancrazio, Anne E. Schaffner, Jeffery L. Barker, David A. Stenger, Central neuronal synapse formation on micropatterned surfaces, Dev. Brain Res. 111 (1998) 231-243.

[13] A. M. Taylor, D. C. Dieterich, H. T. Ito, S. A. Kim, E. M. Schuman, Microfluidic local perfusion chambers for the visualization and manipulation of synapses, Neuron 66(2010) 57-68.

[14] A. Reska, P. Gasteier, P. Schulte, M. Moeller, A. Offenhäusser, J. Groll, Ultrathin coatings with change in reactivity over time enable functional in vitro networks of insect neurons, Adv. Mater. 20 
(2008) 2751-2755.

[15] J. Erickson, A. Tooker, Y. C. Tai, J. Pine, Caged neuron MEA: a system for long-term investigation of cultured neural network connectivity, J. Neurosci. Methods 175(2008) 1-16.

[16] T. Asano, H. Uno, K. Shibasaki, M. Tominaga, T. Urisu, A cell culture type planar ion channel biosensor, Trans. Mater. Res. Soc. Jpn. 33(2008) 767-770.

[17] T. Urisu, T. Asano, Z. L. Zhang, H. Uno, R. Tero, H. Junkyu, H. Isoda, Y. Arima, H. Iwata, K. Shibasaki, M. Tominaga, Incubation type Si-based planar ion channel biosensor, Anal. Bioanal. Chem. 391(2008) 2703-2709.

[18] J. C. Curtis, K. Baldwin, B. J. Dworak, J. Stevenson, E. Delivopoulos, N. K. MacLeod, A. F. Murray, Seal formation in silicon planar patch-clamp microstructures, J. Microelectromech. Syst. 17(2008) 974-983.

[19] C.-Y. Chen, T.-Y. Tu, C.-H. Chen, D.-S. Jong, A. M. Wo, Patch clamping on plane glass-fabrication of hourglass aperture and high-yield ion channel recording, Lab Chip 9(2009) 2370-2380.

[20] T. Sordel, F. Kermarrec, Y. Sinquin, I. Fonteille, M. Labeau, F. Sauter-Starace, C. Pudda, F. de Crécy, F. Chatelain, M. De Waard, C. Arnoult, N. Picollet-D'hahan, The development of high quality seals for silicon patch-clamp chips, Biomaterials 31(2010) 7398-7410.

[21] S. A. Ruiz, C. S. Chen, Microcontact printing: a tool to pattern, Soft Matter 3(2007) 168-177.

[22] G. Nagel, T. Szellas, W. Huhn, S. Kateriya, N. Adeishvili, P. Berthold, D. Ollig, P. Hegeman, E. Bamberg, Channelrhodopsin-2, a directly light-gated cation-selective membrane channel, Proc. Natl. Acad. Sci. USA 100(2003) 13940-13945.

[23] F. Zhang, L.-P. Wang, E. S. Boyden, K. Deisseroth, Channelrhodopsin-2 and optical control of excitable cells, Nat. Methods 3(2006) 785-792.

[24] T. Ishizuka, M. Kakuda, R. Araki, H. Yawo, Kinetic evaluation of photosensitivity in genetically engineered neurons expressing green algae light-gated channels, Neurosci. Res. 54(2006) 85-94.

[25] P. Schoenenberger, A. Grundits, T. Rose, T. G. Oertner, Optimizing the spatial resolution of Channelrhodopsin-2 activation, Brain Cell Biol.36 (2008) 119-127.

[26] C. Bamann, T. Kirsch, G. Nagel, E. Bamberg, Spectral characteristics of the photocycle of channelrhodopsin-2 and its implication for channel function, J. Mol. Biol. 375(2008) 686-694.

[27] H. Wang, Y. Sugiyama, T. Hikima, E. Sugano, H. Tomita, T. Takahashi, T. Ishizuka, H. Yawo, Molecular determinants differentiating photocurrent properties of two channelrhodopsins from Chlamydomonas, J. Biol. Chem. 284(2009) 5685-5696.

[28] B. Y. Chow, X. Han, A. S. Dobry, X. Qian, A. S. Chuong, M. Li, M. A. Henninger, G. M. Belfort, Y. Lin, P. E. Monahan, E. S. Boyden, High-performance genetically targetable optical neural silencing via light-driven proton pumps, Nature 463(2010) 98-102.

[29] L. Wen, H. Wang, S. Tanimoto, R. Egawa, Y. Matsuzaka, H. Mushiake, T. Ishizuka, H. Yawo, 
Opto-current-clamp actuation of cortical neurons using a strategically designed channel rhodopsin, PLoS One 5(2010) e12893.

[30] M.-S. Huang, Y.-C. Chiang, S.-C. Lin, H.-C. Cheng, C.-F. Huang, Y.-K. Shen, Y. Lin, Fabrication of microfluidic chips using micro-hot embossing with micro electrical discharge machining mold, Polym. Adv. Technol. 23(2012) 57-64.

[31] T. Nagai, K. Ibata, E.-S. Park, M. Kubota, K. Mikoshiba, A. Miyawaki, A variant of yellow fluorescent protein with fast and efficient maturation for cell-biological applications, Nat. Biotechnol. 20 (2002) 87-90.

[32] M. J. Caterina, M. A. Schumacher, M. Tominaga, T. A. Rosen, J. D. Levine, D. Julius, The capsaicin receptor: a heat-activated ion channel in the pain pathway, Nature 389 (1997) 816-824.

[33] N. Akaike, N. Harata, Nystatin perforated patch recording and its applications to analyses of intracellular mechanisms, Jpn. J. Physiol. 44(1994) 433-473.

[34] K. V. Snyder, A. M. Kriegstein, F. Sachs, A convenient electrode holder for glass pipettes to stabilize electrode potentials, Pflügers Arch-Eur. J. Physiol. 438 (1999) 405-411.

[35] A. W. Hassel, K. Fushimi, M. Seo, An agar-based silver|silver chloride reference electrode for use in micro-electrochemistry, Electrochem. Commun. 1(1999) 180-183.

[36] X. M. Shao, J. L. Feldman, Micro-agar salt bridge in patch-clamp electrode holder stabilizes electrode potentials, J. Neurosci. Methods 159(2007) 108-115.

[37] M. Mayer, J. K. Kriebel, M. T. Tosteson, G. M. Whitesides, Microfabricated Teflon membranes for low-noise recordings of ion channels in planar lipid bilayers, Biophys. J. 85(2003) 2684-2695.

[38] Z.-H. Wang, N. Takada, H. Uno, T. Ishizuka, H. Yawo, T. Urisu, Positioning of the sensor cell on the sensing area using cell trapping pattern in incubation type planar patch clamp biosensor, Colloids Surf. B Biointerfaces 96 (2012) 44-49.

[39] L. Vyklický, K. Nováková-Toušová, J. Benedikt, A. Samad, F. Touška, V. Vlachová, Calcium-dependent desensitization of vanilloid receptor TRPV1: a mechanism possibly involved in analgesia induced by topical application of capsaicin, Physiol. Res. 57 (2008) S59-S68.

[40] K. Stehfest and P. Hegemann, Evolution of the Channelrhodopsin Photocycle Model, ChemPhysChem 11 (2010) 1120-1126.

[41] Yugo Fukazawa, Ryuichi Shigemoto, Intra-synapse-type and inter-synapse-type relationships between synaptic size and AMPAR expression, Curr. Opin. in Neurobiol., 22 (2012) 1-7.

[42] B. Scelfo, M. Politi, F. Reniero, T. Palosaari, M. Whelan, J-M. Zaldivar, Application of multielectrode array (MEA) chips for the evaluation of mixtures neurotoxicity, Toxicology 299 (2012) 172-183.

[43] Q.-Y. Liu, M. Coulombe, J. Dumm, K. M. Shaffer, A. E. Schaffner, J. L. Barker, J. J. Pancrazio, D. A. Stenger, W. Ma, Synaptic connectivity in hippocampal neuronal networks cultured on 
micropatterned surfaces, Dev. Brain Res., 120 (2000) 223-231.

[44] H.-Z.W. Tao, L. I. Zhang, G.-Q. Bi, M.-M. Poo, Selective presynaptic propagation of long-term potentiation in defined neural networks, J. Neurosci. 20 (2000) 3233-3243.

[45] K. S. Wilcox, J. Buchhalter, M. A. Dichter, Properties of Inhibitory and excitatory synapses between hippocampal neurons in very low density cultures, SYNAPSES 18 (1994) 128-151. 


\section{Biographies}

Hidetaka Unois a Researcher atNagoya University, Research Center for Innovative Nanobiodevices.

He obtained a PhD degree from the Graduate University for Advanced Studies in 2012.

Zhi-hong Wangis an Assistant Professorat Nagoya University, Research Center for Innovative Nanobiodevices. He obtained a PhD degree from the Graduate University for Advanced Studies in 2003.

Ysutaka Nagaokais a Researcher at Nagoya University, Research Center for Innovative Nanobiodevices. He obtained a PhD degree from the University of Tokyo in 2010.

Noriko Takadais aTechnical staff at the Institute for Molecular Science. Her interest is microfabrication technology, especially photolithography.Shereceived a Master's degree from the Faculty of Agriculture, Tokyo University of Agriculture and Technology Japan in 2004.

SenthilkumarObulirajis an Assistant Professor in the Department of Chemistry,Ramco Institute of Technology,India. He obtained a PhD degree from Anna University India in 2005. His interest is in semiconductor processes.

Kei Kobayashi is a Researcher at Nagoya University, Research Center for Innovative Nanobiodevices. He obtained a PhD degree from the Tokyo Institute of Technology in 1999. His interest is in molecular biology.

Toru Ishizuka is a Lecturer at Tohoku University, Graduate School of Life Sciences. He received a PhD degree from Niigata University in 1997. His interests cover molecular and cellular neuroscience.

Hiromu Yawois a Professor atTohoku University, Graduate School of Life Sciences. He received a PhD degree from Kyoto University in 1981. His interests cover molecular and cellular neuroscience.

Tsuneo Urisuis a Professor at Nagoya University, Research Center for Innovative Nanobiodevices.He received a PhD from the University of Tokyoin 1972. His interests cover neural network devices and their applications .

Yukio Komatsuis a Professor at Nagoya University, Research Institute of Environmental Medicine. He received a $\mathrm{PhD}$ from Nagoya University in 1983. His interests cover cellular and system neuroscience. 


\section{Figure Captions}

Fig. 1 (a) Schematic diagram showing the ion channel biosensor a.

Fig. 2 (a) Schematic diagram and photograph of the $\mathrm{AgCl} / \mathrm{Ag}$ stable electrode with a salt bridge structure, and (b) Comparison of the baseline fluctuations in the simple $\mathrm{AgCl} / \mathrm{Ag}$ electrode and the salt bridge-type stable electrode.

Fig. 3Structures of the sensor chip. (a) Back side view of the Si sensor chip. The micropore formed by FIB milling is shown. (b) Cross-sectional structure of the sensor chip. The cell trapping area was formed by photolithography in Si and hot embossing in PMMA. (c) Scanning electron microscopy images of the Ni mold used to form the cell trapping pattern by hot embossing. The cell trapping areas were formed at all crossing points of the lattice pattern. On the other hand, the micropore was formed only in the cell trapping area at the center of the chip. The scale barindicates $100 \mu \mathrm{m}$. (d) Optical microscopy image of the cell trapping area of the PMMA sensor chip. The micropore formed by FIB milling isshown. The cross section with line A-B is shown in (b). The scale bar indicates $10 \mu \mathrm{m}$.

Fig. 4(a)Optical microscopy view of the Sisensor chip with a flat surface after 5 days incubation of TRPV1-expressing HEK293 cells seeded at 200 cells $/ \mathrm{mm}^{2}$. (b) Fluorescence microscopy image of the ChRWR-expressing HEK293 cells after 3 days incubation on a PMMA sensor chipprepared with a cell trapping pattern.The fluorescence from bright Venus [31]is observed. The cell on the micropore is indicated by the dotted line. The scale bar indicates $50 \mu \mathrm{m}$.

Fig. 5 (a)Capsaicin-stimulated ion channel current recordings of the TRPV1 expressed on the HEK293 (Fig. 4 a), in the presence (upper) and absence (lower) of $\mathrm{Ca}^{2+}$ ions. The capsaicin concentration was 1 $\mu \mathrm{M}$. (b) The dependence of the channel current on the capsaicin concentration. The membrane potential was $-30 \mathrm{mV}$ in both (a) and (b).

Fig. 6(a) Channel current recordings of ChRWR expressed on the HEK293 cells (Fig.4 b) after stimulation by laser illumination $(\lambda=473 \mathrm{~nm}$, output power $=1.5 \mathrm{~mW})$. The membrane potentials were -90 $\mathrm{mV},-60 \mathrm{mV},-30 \mathrm{mV}, 30 \mathrm{mV}, 60 \mathrm{mV}$, and $90 \mathrm{mV}$, from the bottom to the top panels. (d) The dependence of the channel current on the membrane potential.

Fig. 7 Spontaneous channel current measurements in the in vitro rat hippocampus neuron network. (a) Bright field image of the neuron network formed over 14 days of culturingon the Si sensor chip prepared with a flat surface. Micropore position (arrow mark) was identified using back-light illuminations. Dotted 
line shows single nerve cell on the micropore. (b) Spontaneous channel currents observed for several membrane potentials. Effects of the addition of TTX were investigated for a membrane potential of -40 $\mathrm{mV}$ and $+40 \mathrm{mV}$. The smooth line around the center indicates the recording obtained at a membrane potentialof $-30 \mathrm{mV}$ prior to nystatin injectioninto the pipette solution. All other recordings were measured after nystatin injection. 
Figure 2

(a)

(b)

$\mathrm{AgCl} / \mathrm{Ag}$

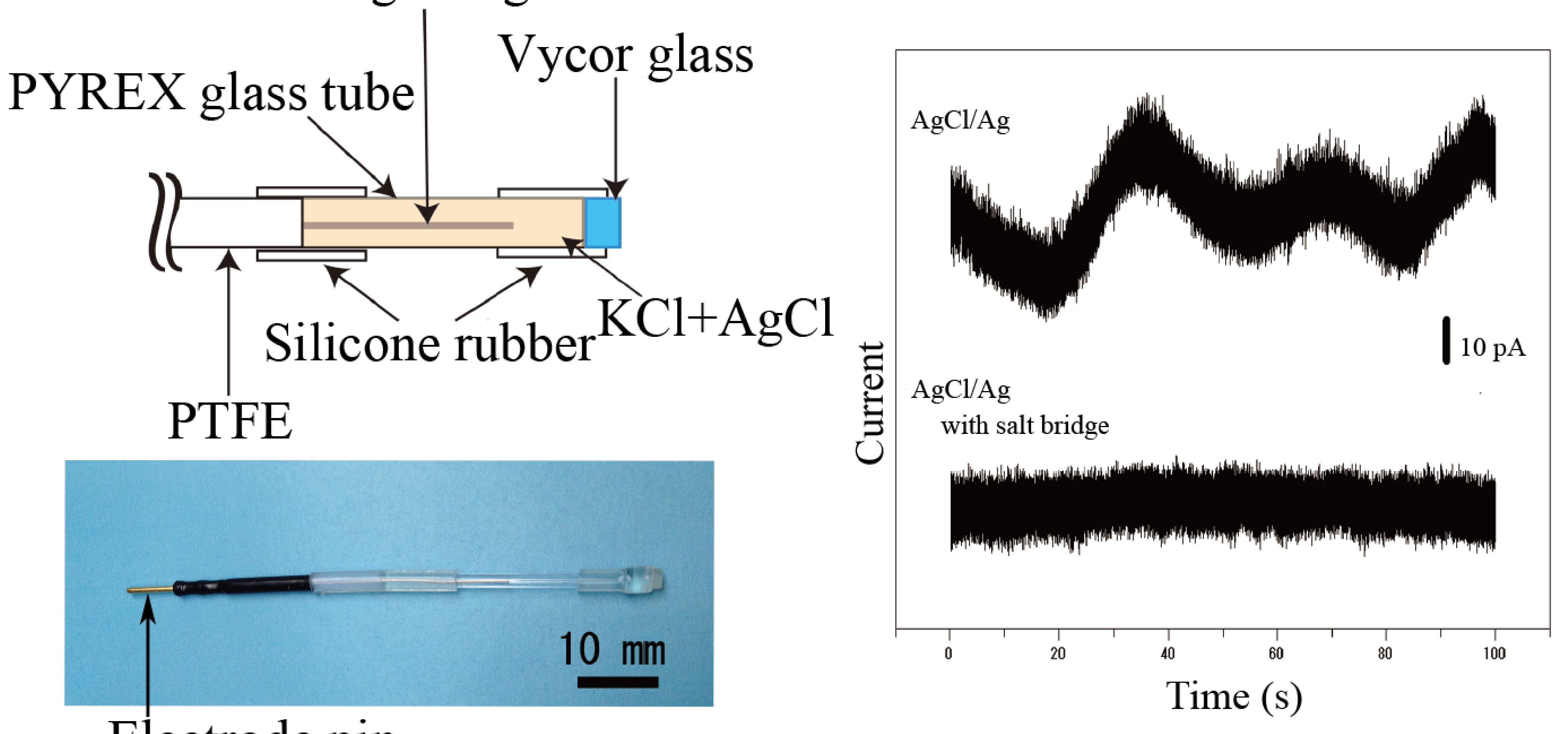

Electrode pin 
(a)

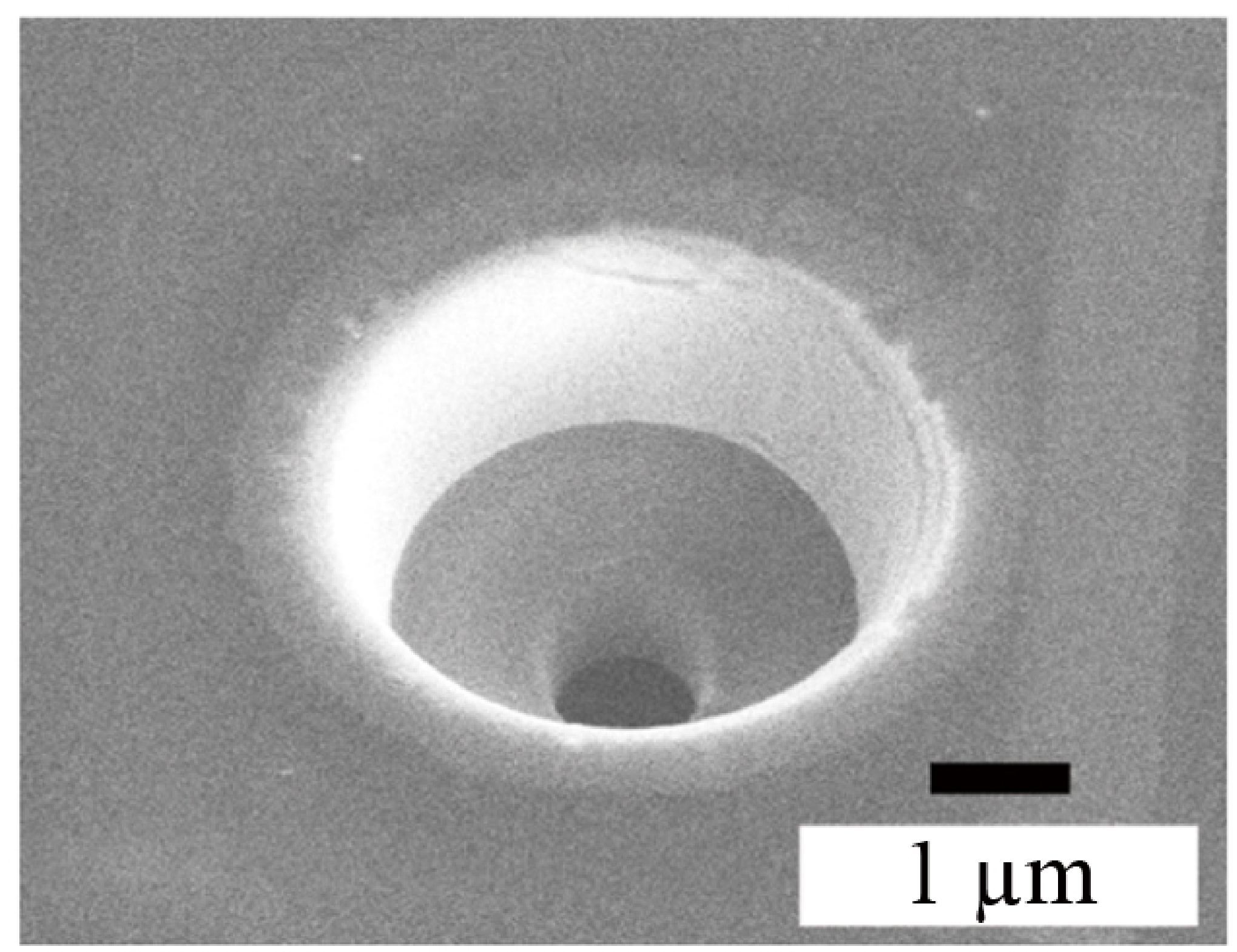

(c)

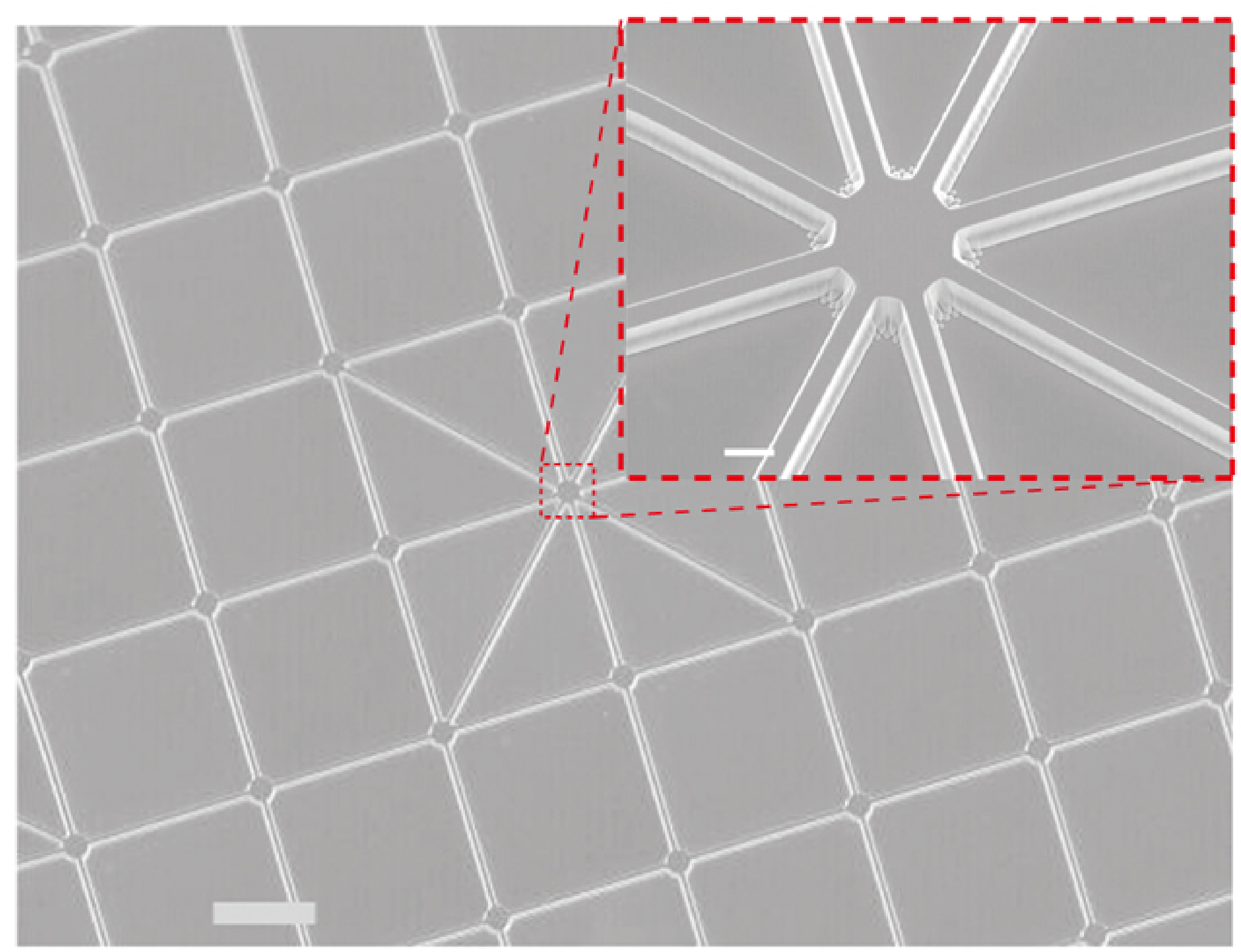

(b)

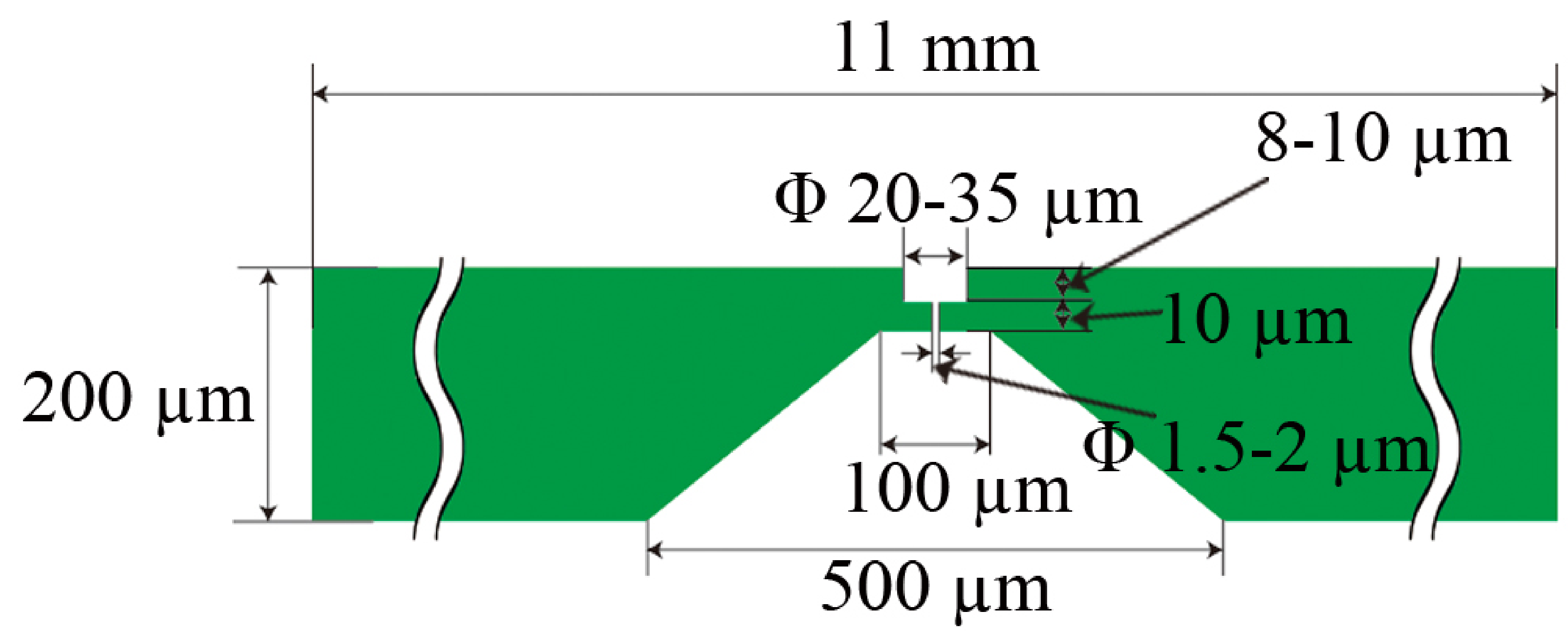

(d)

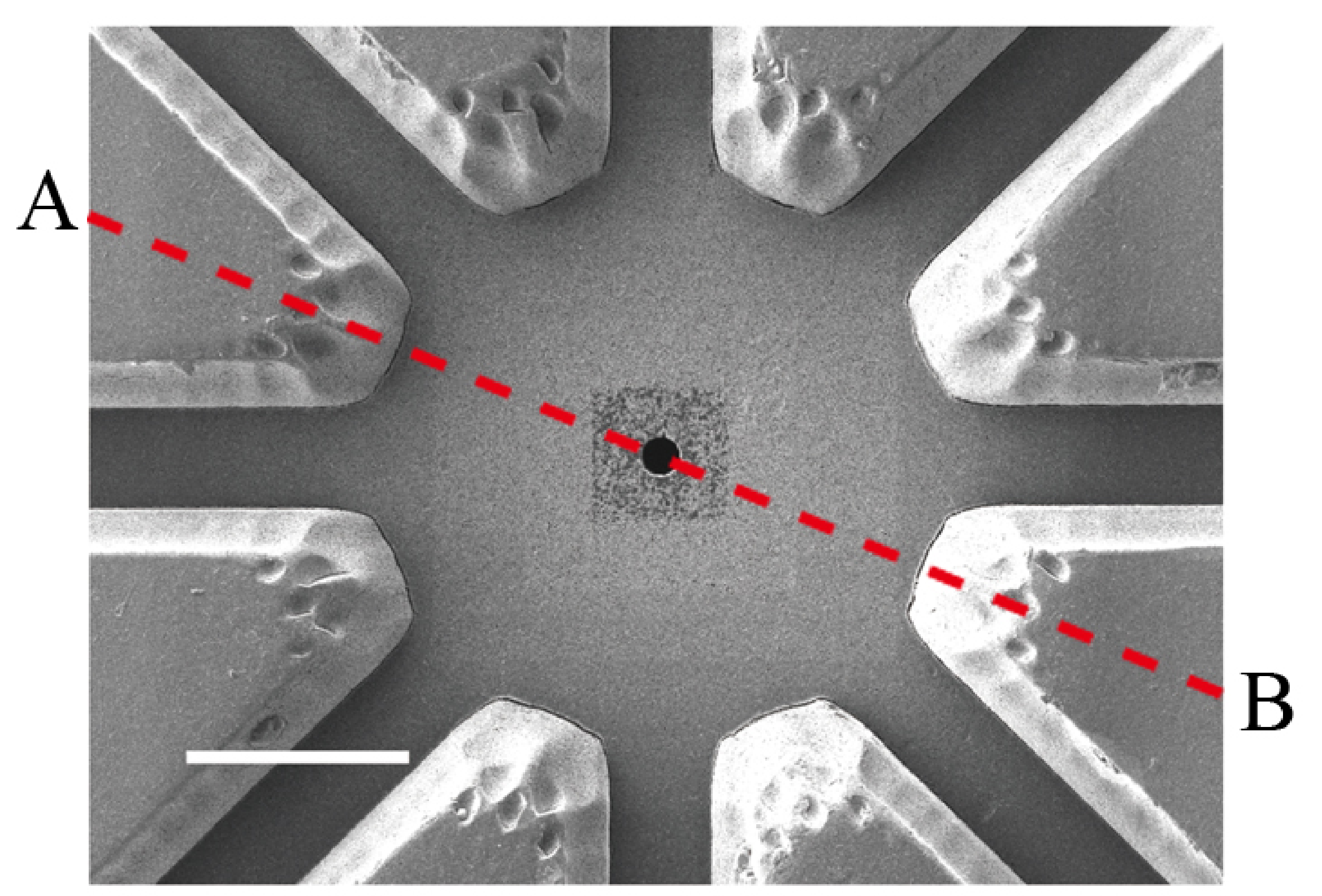



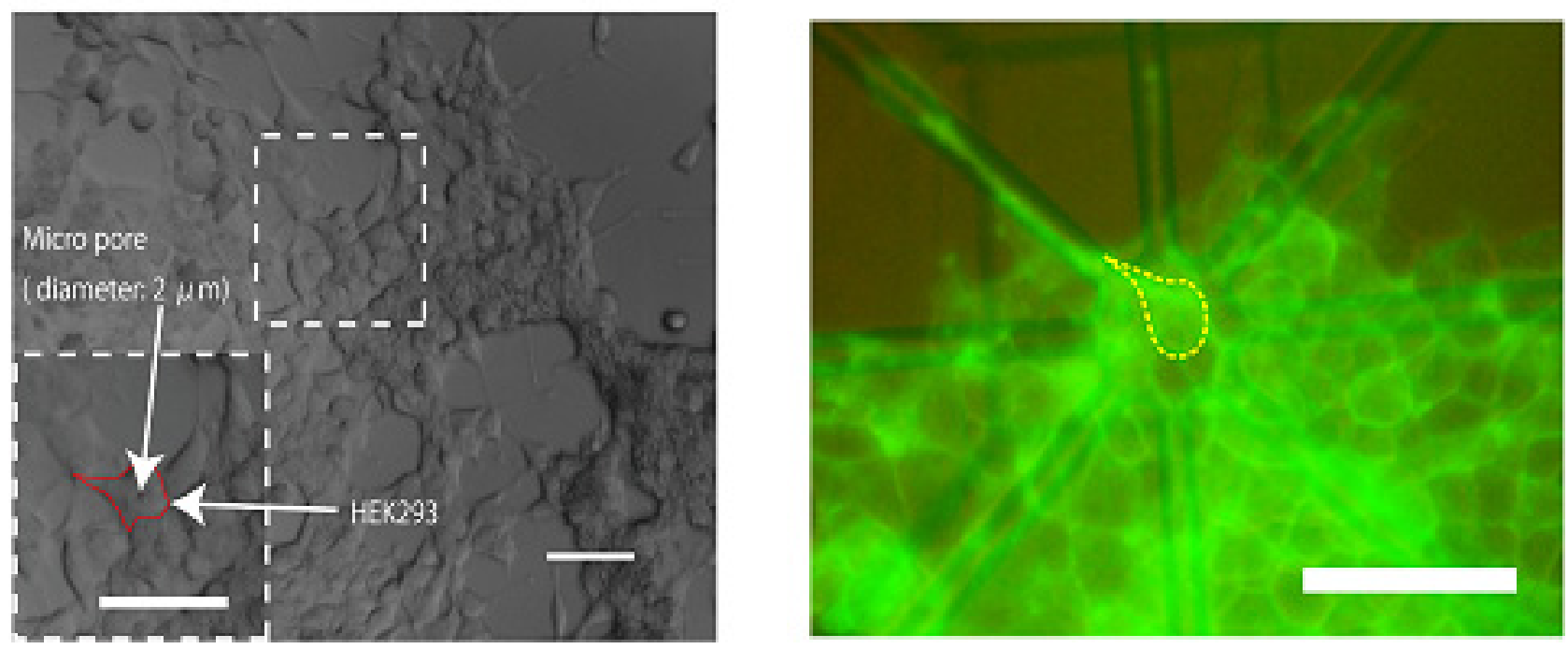

Fig. 4 
(a)

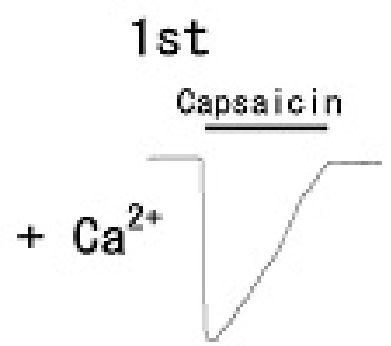

2nd

Capsaicin

$0.5 \frac{\mathrm{nA}}{5 \mathrm{~s}}$

3rd

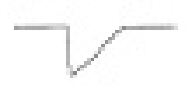

Capsaicin

Capsaicin

Capsaicin

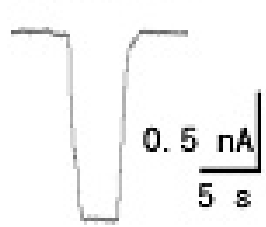

(b)

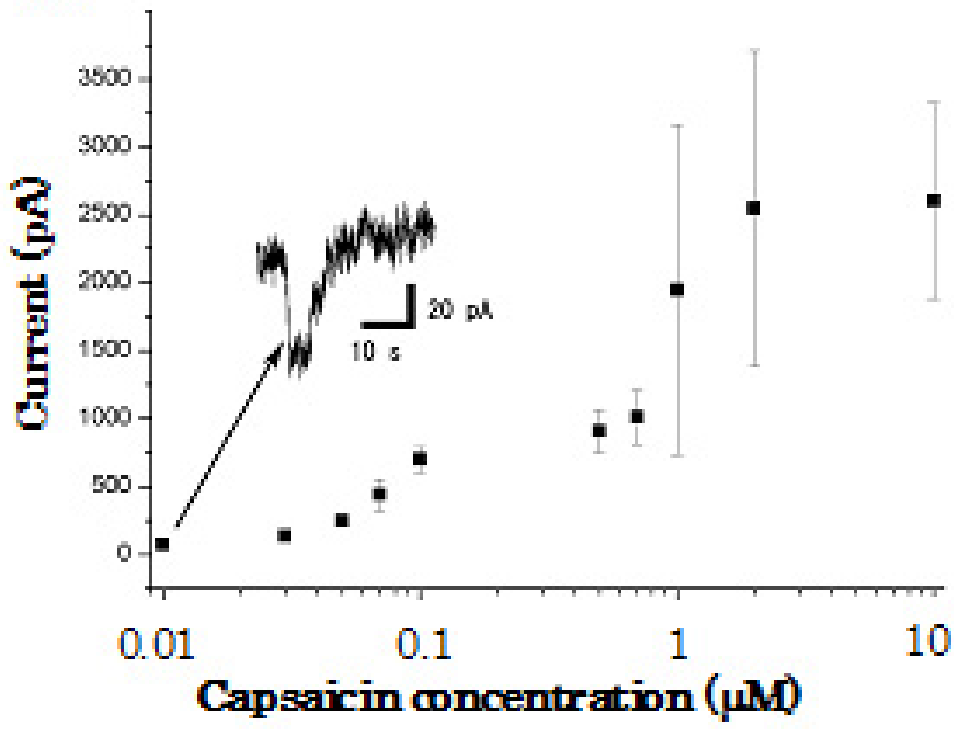

Fig. 5 


\section{(a)}

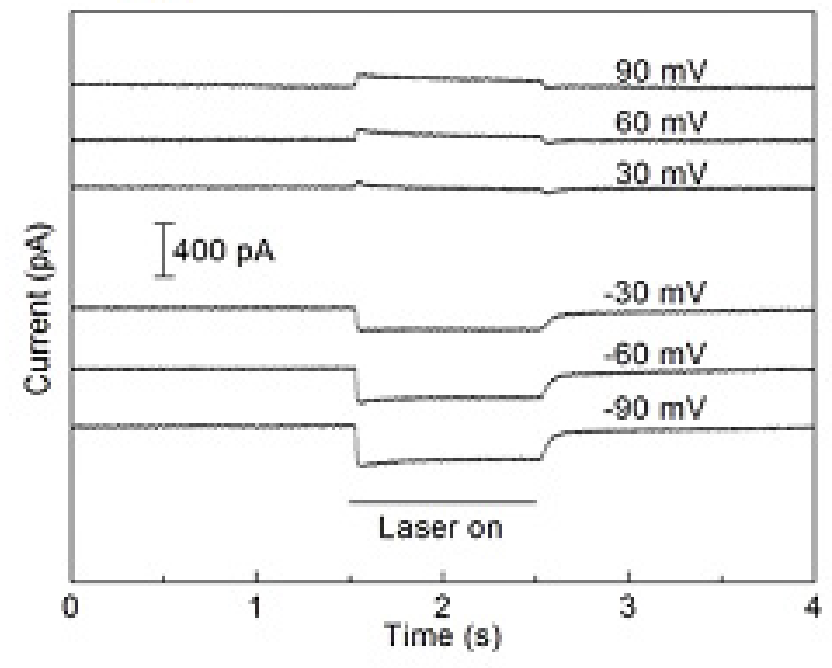

\section{(b)}

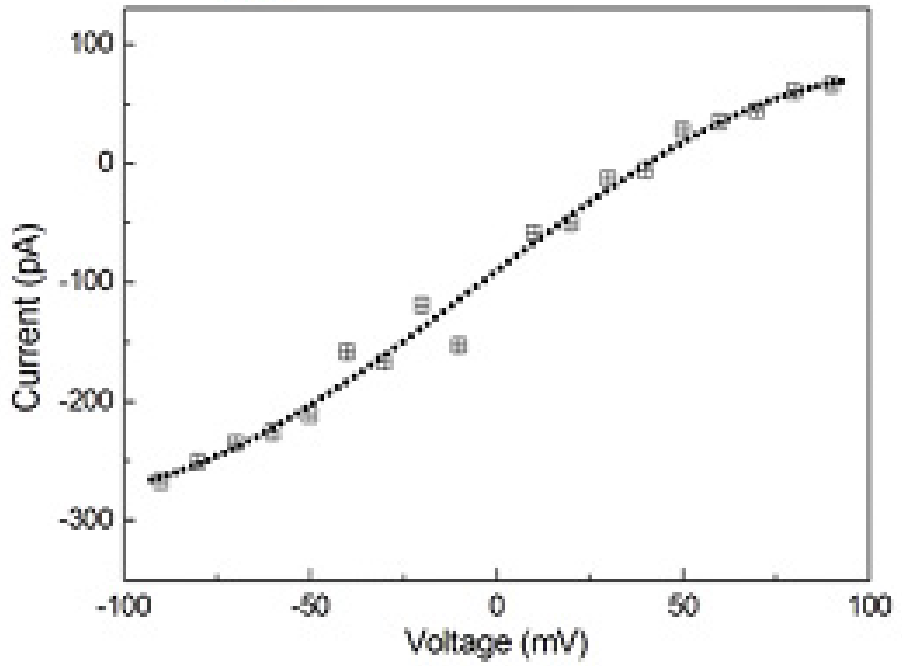

Fig. 6 
Figure 7

(a)

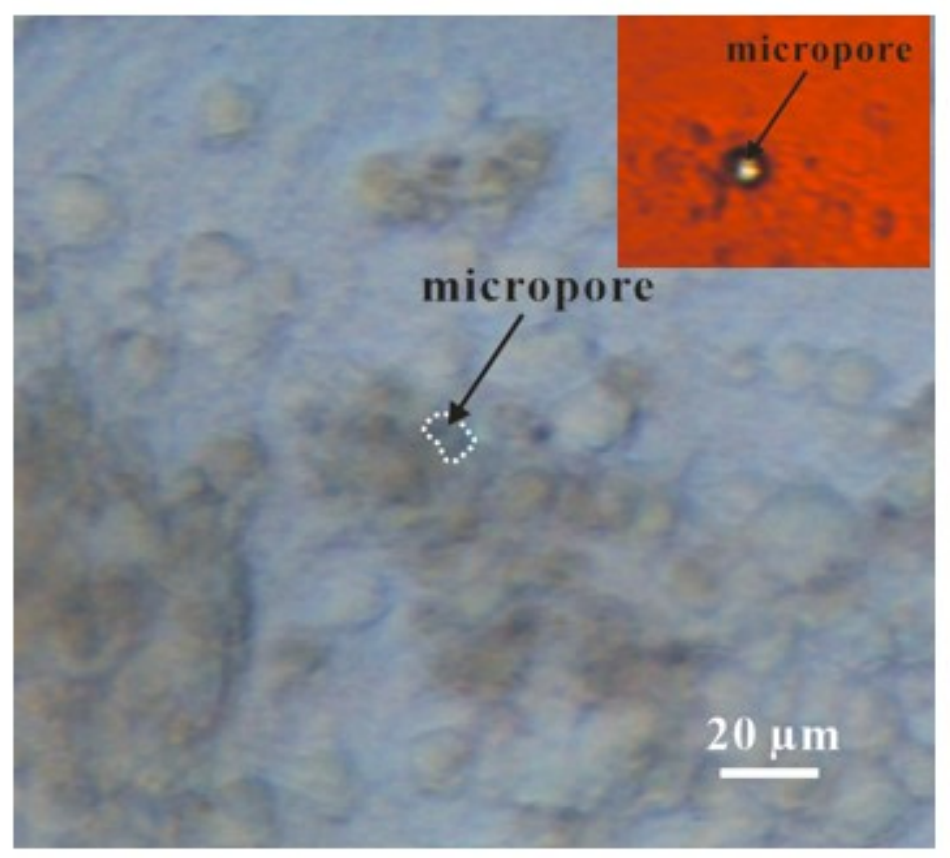

(b)

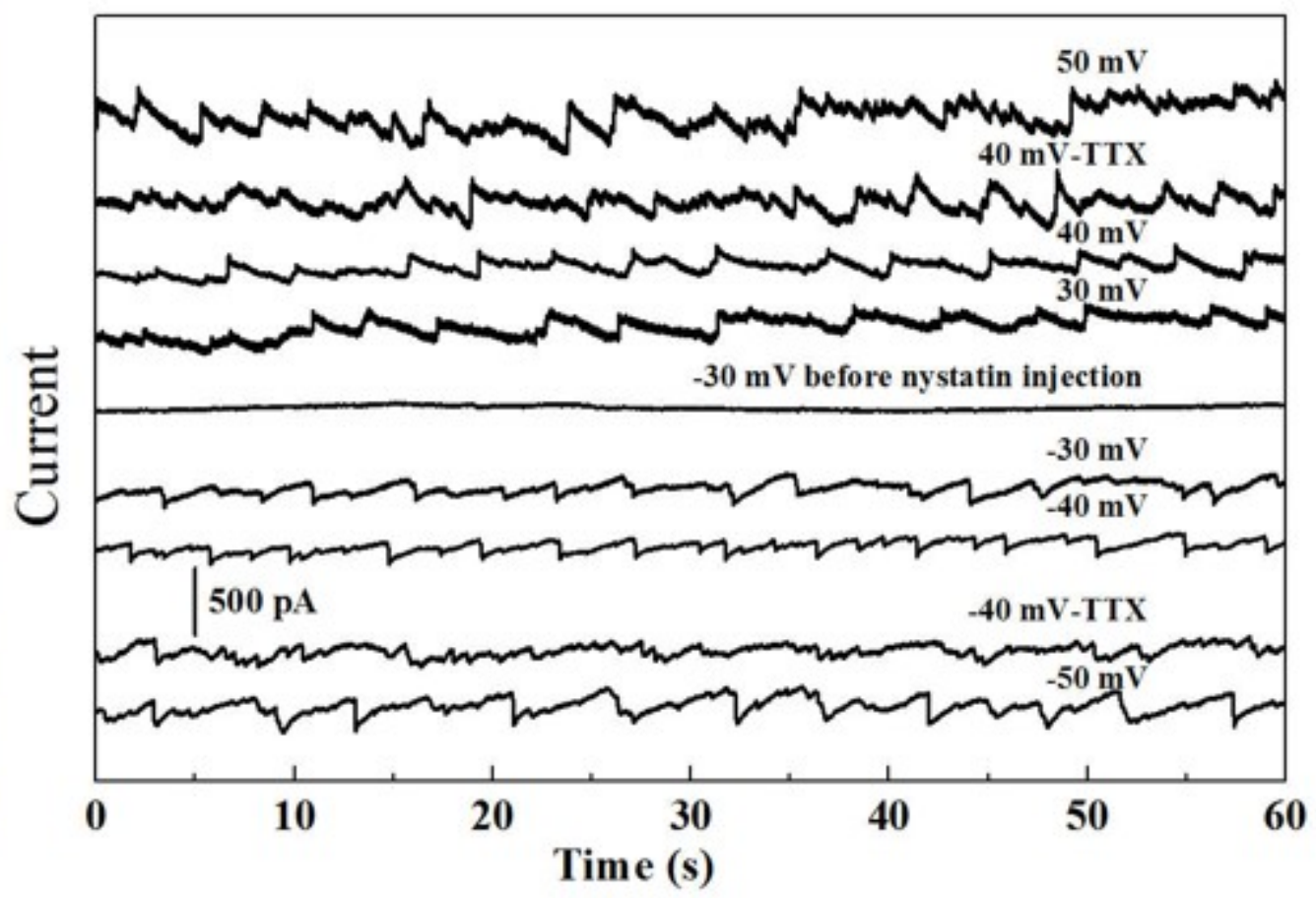

